\title{
The nuclear translocation of transketolase inhibits the farnesoid receptor expression by promoting the binding of HDAC3 to FXR promoter in hepatocellular carcinoma cell lines
}

\author{
Minle Li ${ }^{1,2}$, Xuping Zhang ${ }^{1,2}$, Ying $\mathrm{Lu}^{3}$, Sen Meng ${ }^{1,2}$, Haoyu Quan ${ }^{1,2}$, Pingfu Hou ${ }^{1,2}$, Pan Tong ${ }^{4}$, Dafei Chai ${ }^{1,2}$, \\ Xiaoge Gao ${ }^{1,2}$, Junnian Zheng ${ }^{1,2,5}$, Xuemei Tong ${ }^{3}$ and Jin Bai, ${ }^{1,2}$
}

\begin{abstract}
Transketolase (TKT), which is a metabolic enzyme in the nonoxidative phase of the pentose phosphate pathway (PPP), plays an important role in providing cancer cells with raw materials for macromolecular biosynthesis. The ectopic expression of TKT in hepatocellular carcinoma (HCC) was reported previously. However, the role of TKT in the initiation of liver cancer is still obscure. In our previous study, we found that TKT deficiency protects the liver from DNA damage by increasing levels of ribose 5-phosphate and nucleotides. What's more interesting is that we found TKT deficiency reduced bile acids and loss of TKT promoted the farnesoid receptor (FXR) expression. We further showed that TKT translocated into the nucleus of HCC cell lines through interacting with the signal transducer and activator of transcription 1 (STAT1), and then the complex inhibited FXR expression by promoting the binding of histone deacetylase 3 (HDAC3) to FXR promoter.
\end{abstract}

\section{Introduction}

Hepatocellular carcinoma (HCC) is the fifth most common tumor in adults ${ }^{1}$ and is a major cause of cancer death worldwide ${ }^{2}$. Despite continuous improvement in treatment over the past decades, the five-year survival rate of HCC is still less than $10 \%^{3,4}$. The lack of effective treatment is one of the main reasons for the high mortality of HCC. Therefore, exploring the mechanisms behind the occurrence of HCC and identifying effective target genes are particularly important for the prevention and treatment of HCC.

Correspondence: Junnian Zheng (jnzheng@xzhmu.edu.cn) or

Xuemei Tong (xuemeitong@shsmu.edu.cn) or Jin Bai (bj@xzhmu.edu.cn)

${ }^{1}$ Cancer Institute, Xuzhou Medical University, 221002 Xuzhou, Jiangsu, China

${ }^{2}$ Jiangsu Center for the Collaboration and Innovation of Cancer Biotherapy, Xuzhou Medical University, 221002 Xuzhou, Jiangsu, China

Full list of author information is available at the end of the article.

These authors contributed equally: Minle Li, Xuping Zhang, Ying Lu

Edited by G. Ciliberto
Some studies have shown that the metabolism of bile acids in liver is closely related to the occurrence of liver cancer. Bile acids are not only physiological detergent molecules synthesized from cholesterol in the liver, but also they are highly cytotoxic ${ }^{5}$. Under normal physiological conditions, bile acid secretion generates bile flow and promotes hepatobiliary secretion of various endogenous metabolites and xenobiotics to increase cholesterol solubility and decrease bile acid toxicity ${ }^{6}$. However, impaired bile flow can lead to cholestasis, and then accumulation of bile acids in the liver causes hepatic inflammation and injury ${ }^{7}$. Studies have shown that cholestasis is related to fibrosis, cirrhosis, and eventually the development of hepatocellular carcinoma.

The farnesoid receptor (FXR) belongs to the nuclear receptor family and is highly expressed in hepatocytes and intestine ${ }^{8}$. Recent studies have shown that FXR not only participates in the regulation of bile acids ${ }^{9,10}$ but also plays an important role in regulating hepatic fibrosis, 
cholestasis, and inflammation ${ }^{11-13}$. It has been shown that FXR inhibits the absorption of hepatic bile acid and promotes bile acid secretion by promoting the expression of the hepatic bile acid transporter Bile Salt Export Pump (BSEP), which prevents bile acid accumulation in hepatocytes $^{14}$. Some studies have reported that FXR knockout mice develop spontaneous liver tumors ${ }^{15,16}$.

TKT is one of the metabolic enzymes in the nonoxidative phase of the pentose phosphate pathway, which plays an important role in promoting the rapid proliferation of tumor cells. TKT-null mice die in embryo and disruption of one TKT allele can cause growth retardation ${ }^{17}$. Emerging evidence also showed that in cervical and pancreatic cancer, high expression of TKT provides raw materials for DNA and RNA synthesis in tumor cells by enhancing the activity of $\mathrm{PPP}^{18,19}$. The ectopic expression of TKT in HCC has been reported previously ${ }^{4,20}$. However, the roles of TKT in the initiation of liver cancer are still obscure. In our previous study, we generated a liver-specific TKT knockout mouse strain, we found a novel role of TKT in increasing genome instability by limiting de novo nucleotide biosynthesis, and loss of the enzyme significantly reduced liver injury and cancer initiation ${ }^{21}$. In addition, metabolomic analysis showed that TKT deficiency reduced bile acids in livers. We further showed that TKT entered the nucleus with the help of STAT1 to inhibit FXR expression through promoting the binding of HDAC3 to FXR promoter. Taken together, our findings may provide a novel metabolic target for HCC prevention.

\section{Materials and methods}

\section{Mice}

The $T K T^{f l / f l} A l b$-cre mice in $\mathrm{C} 57 \mathrm{BL} / 6$ genetic background were kindly provided by Dr. Xuemei Tong at Shanghai Jiao Tong University School of Medicine. Animals were maintained under specific pathogen-free conditions and all experiments were conducted in accordance with the guidelines for animal care at the Shanghai Jiao Tong University School of Medicine. In all animal studies, the sample size is more than three pairs.

\section{Antibodies and reagents}

The specific antibodies used in this study were as follows: anti-TKT (8616, Cell Signaling Technology; Abcam, 112997), anti-STAT1 (14994, Cell Signaling Technology), anti-Tubulin (10004185, Proteintech) and anti-PARP (GTX20833, GeneTex), anti-FXR (ab28480, Abcam; 25055-1-AP, Proteintech).

\section{Mouse models of liver cancer and analysis}

In the DEN-induced HCC model, DEN $(25 \mathrm{mg} / \mathrm{kg}$ ) was injected i.p. into 2-week male mice once. Starting at 4 weeks of age, the mice were fed a high-fat diet until they were sacrificed at 6 or 9 months of age. Their livers were removed and separated into different lobes. Visible tumors were counted and measured.

\section{Mass spectrometry of bile acids in livers}

Liver $(30 \mathrm{mg}$ ) was removed from WT/KO mouse, was added with pre-cold $80 \%$ (vol/vol) methanol to extract metabolites followed by centrifugation at $16,000 \times g$ for $10 \mathrm{~min}$ at $4{ }^{\circ} \mathrm{C}$. The supernatant was dried with $\mathrm{N} 2$ at room temperature. The extracts were reconstituted with $100 \mu \mathrm{L} \mathrm{50 \%} \mathrm{(v/v)} \mathrm{methanol} \mathrm{solution.} \mathrm{UHPLC-MS} \mathrm{were}$ performed using an HPLC (Dionex 3000 Ultimate)/ MS/ MS (TSQ Vantage, Thermo Scientific). A $5 \mu \mathrm{L}$ of sample was injected for each analysis. The chromatographic column $(100 \times 2.1 \mathrm{~mm}, 1.9 \mu \mathrm{m}$, Hypersil Gold $)$ was used for separation at $45^{\circ} \mathrm{C}$. Mobile phase A is water with $10 \mathrm{mM}$ ammonium acetate, and mobile phase B is $100 \%$ acetonitrile. The flow rate is $0.4 \mathrm{~mL} / \mathrm{min}$ and gradient program began with $20 \%$ B to $23 \%$ B in 3 min, $23 \%$ B to $32 \%$ B in $5 \mathrm{~min}, 32 \% \mathrm{~B}$ to $80 \% \mathrm{~B}$ in $6 \mathrm{~min}$, and hold on $80 \% \mathrm{~B}$ for $6 \mathrm{~min}$. The instrument was operated in selected reaction monitoring (SRM) and positive ionization mode. The mass analyzer settings were as following: vaporizer temperature $\left(350^{\circ} \mathrm{C}\right)$, spray voltage $(3000 \mathrm{~V})$, capillary temperature $\left(330^{\circ} \mathrm{C}\right)$, sheath gas pressure $(40 \mathrm{arb})$, Aux gas pressure (10 arb), collision gas pressure (mTorr):1.5, Q1 peak width (FWHM): 0.7, Q3 peak width (FWHM): 0.7, cycle time (s): $0.4 \mathrm{~s}$. The SRM parameters of dansyl derivatives of amino acids and IS (d3-proline) are shown in Supplementary Table 1 . The results were corrected with internal standard, and each liver sample is tested three times, and the group with the largest deviation is removed, and the average value is calculated.

\section{Cell culture}

The human cell lines SMMC-7721, HepG2, and L-O2 were purchased from the Shanghai Institute of Biochemistry and Cell Biology, Chinese Academy of Science (Shanghai, China). SMMC-7721, HepG2, and L-O2 cells were cultured in DMEM medium containing 10\% FBS. All cells were maintained in a $5 \% \mathrm{CO}_{2}$ atmosphere at $37^{\circ} \mathrm{C}$.

\section{Western blot analysis}

Total protein extract was obtained using RIPA lysis buffer (Sigma, St. Louis, MO, USA). The protein concentration was measured by the BCA Protein Assay Kit (Pierce, Rockford, IL, USA). Samples were subsequently resolved on 7.5 and 15\% SDS-PAGE gels. Proteins were transferred to Immuno-Blot PVDF Membranes (Bio-Rad, Hercules, CA, USA) and the membranes were blocked in $5 \%$ nonfat milk in Tris buffered saline containing Tween20 . The membrane was incubated with the stated primary antibodies followed by secondary peroxidase labeled antirabbit or anti-mouse antibodies (Santa Cruz, CA, USA). 
The signals were developed using an enhanced chemiluminescent solution (Millipore, Boston, MA, USA).

\section{RNA isolation, reverse-transcription, and real-time quantitative RT-PCR}

Total RNA was extracted with TRIzol (Invitrogen Life Technologies, Carlsbad, CA, USA) according to the manufacturer's instructions. Total RNA was reverse transcribed into cDNA using the PrimeScriptTM RT Reagent Kit (Takara Bio Inc., Shiga, Japan). The SYBR ${ }^{\circledR}$ green Premix Ex TaqTM kit (Takara Bio Inc., Shiga, Japan) was used for real-time PCR analysis, which was performed using an ABI 7500 Fast Sequence Detector (Applied Biosystems, Carlsbad, CA, USA).

\section{ChIP assay}

ChIP assays were performed according to the protocol of the ChIP assay kit (Upstate Biotechnology). SMMC7721 cells cultured in a $100 \mathrm{~mm}$ dish (approximately $1 \times$ $10^{7}$ ) were cross-linked by adding formaldehyde to a final concentration of $1 \%$ and incubated at room temperature for $10 \mathrm{~min}$, washed twice with cold PBS containing protease inhibitors, lysed in ChIP lysis buffer, then sonicated to shear the DNA at $4{ }^{\circ} \mathrm{C}$ to reduce its average length. The sheared DNA was incubated with antibodies against Flag or IgG. DNA-protein-antibody complexes were incubated with ChIP beads with protein A/G (Merck Millipore). The beads were washed with gradients of salt buffer and eluted in $1 \% \mathrm{SDS} / \mathrm{NaHCO}_{3}$. ChIP DNA was analyzed by qRTPCR with primers amplifying the putative regions. The primers sequence are presented in Supplementary Table 2.

\section{Luciferase reporter assay}

SMMC-7721 cells were transfected, and $48 \mathrm{~h}$ later, the cells were harvested and assayed using a luciferase reporter assay system (Promega, USA) according to the manufacturers' instructions.

\section{Coimmunoprecipitation}

Cells were freshly lysed in lysis buffer ( $1 \mathrm{mM}$ EDTA, $40 \mathrm{mM}$ Tris- $\mathrm{HCl}, \mathrm{pH} 8,100 \mathrm{mM} \mathrm{NaCl}, 0.5 \% \mathrm{NP}-40,1 \%$ Triton X-100), and incubated with primary antibodies at $4{ }^{\circ} \mathrm{C}$ overnight, followed by an additional $2-\mathrm{h}$ incubation with protein A/G-agarose beads (Santa Cruz Biotechnology, USA) at $4{ }^{\circ} \mathrm{C}$. The beads were washed with the lysis buffer and boiled in $2 \times$ SDS protein loading buffer. Western blot analysis was performed after immunoprecipitation.

\section{Statistical analysis}

All experiments shown were replicated three times at least. Statistical analysis was performed using Prism 5 (GraphPad Software, San Diego, CA, USA). All reported $P$ values were two-sided, and $P<0.05$ was considered to be statistically significant.

\section{Results \\ TKT deficiency reduced bile acids in livers}

To investigate the protective role of TKT deficiency during liver carcinogenesis, we performed metabolomic analysis. Surprisingly, we found that in the livers of $T K T^{f / f l}$ Alb-Cre mice, primary and secondary bile acids were decreased compared with $T K T^{+/+}$Alb-cre mice (Fig. 1a). Then, we performed mass spectrometry in livers and serum from mice following DEN/HFD treatment for 6 months, and the results further confirmed that TKT deficiency reduced bile acids in livers (Fig. 1b, c).

To determine the role of TKT in bile acid metabolism, we detected the level of FXR. QPCR confirmed that loss of TKT could induce the expression of FXR and its target gene BSEP at the mRNA level (Fig. 1d). These results suggested that TKT might regulate FXR expression to affect bile acid level in the liver.

\section{The expression of FXR is negatively controlled by TKT}

To determine the relationship between TKT and FXR, we changed TKT expression in normal liver cells L-O2 and two different HCC cell lines, SMMC-7721 and HepG2. Three independent shRNA sequences (TKTshRNA-1, TKT-shRNA-2, and TKT-shRNA-3) profoundly suppressed TKT expression in L-O2, SMMC7721 and HepG2. We found that knockdown of TKT increased FXR protein and mRNA levels (Fig. 2a, c, e, and $\mathrm{g}$ ). In contrast, the overexpression of TKT suppressed FXR production in L-O2, SMMC-7721, and HepG2 (Fig. 2b, d, f and h). These results indicated that TKT regulated FXR expression negatively.

\section{TKT translocated into the nucleus and regulated the activity of the FXR promoter}

Given that TKT negatively regulated FXR expression, we hypothesized that TKT may translocate into the nucleus and regulate the activity of the FXR promoter. To verify our hypothesis, a luciferase reporter assay was conducted in SMMC-7721 cells. We found that overexpression of TKT inhibited the activity of the FXR promoter (Fig. 3a). This result indicates that TKT might be located in the nucleus. Then, we analyzed endogenous TKT subcellular localization using both cell fraction analysis and immunofluorescent staining. Subcellular fractionation showed that TKT was present in both the cytosolic and nuclear fractions (Fig. 3b). Immunofluorescent staining using an anti-TKT antibody confirmed that TKT could be located in the nucleus (Fig. 3c), and immunofluorescent staining using an anti-Flag antibody confirmed that overexpressed TKT could be located in the nucleus (Supplementary Fig. S1). Therefore, TKT 


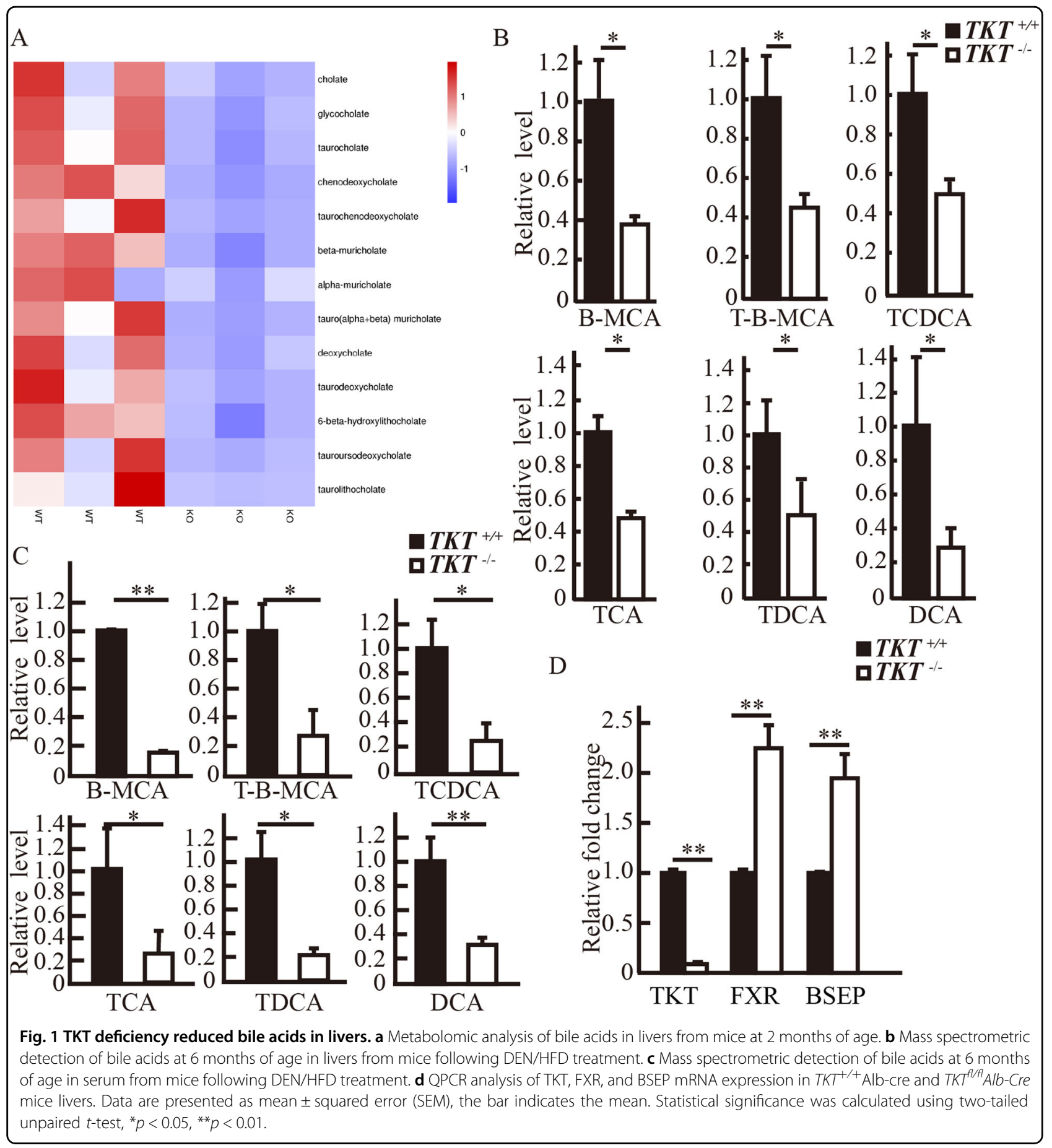

can be present in the nucleus where it may regulate the FXR promoter.

\section{TKT translocated into the nucleus through interacting with STAT1}

Next, we studied how does TKT translocate into the nucleus. We found that TKT had no obvious nuclear location sequence, so we suspected that TKT needs the help of other proteins to enter the nucleus. To find the cofactor, we immunoprecipitated SMMC-7721 cell lysates using the Flag antibody and IgG followed by SDS-PAGE and mass spectrometry analysis. Among the TKT immunoprecipitates, we focused on STAT1 because it can enter the nucleus (Fig. 4a). Then, we verified the interaction between STAT1 and TKT using coimmunoprecipitation. We confirmed that TKT coimmunoprecipitated 


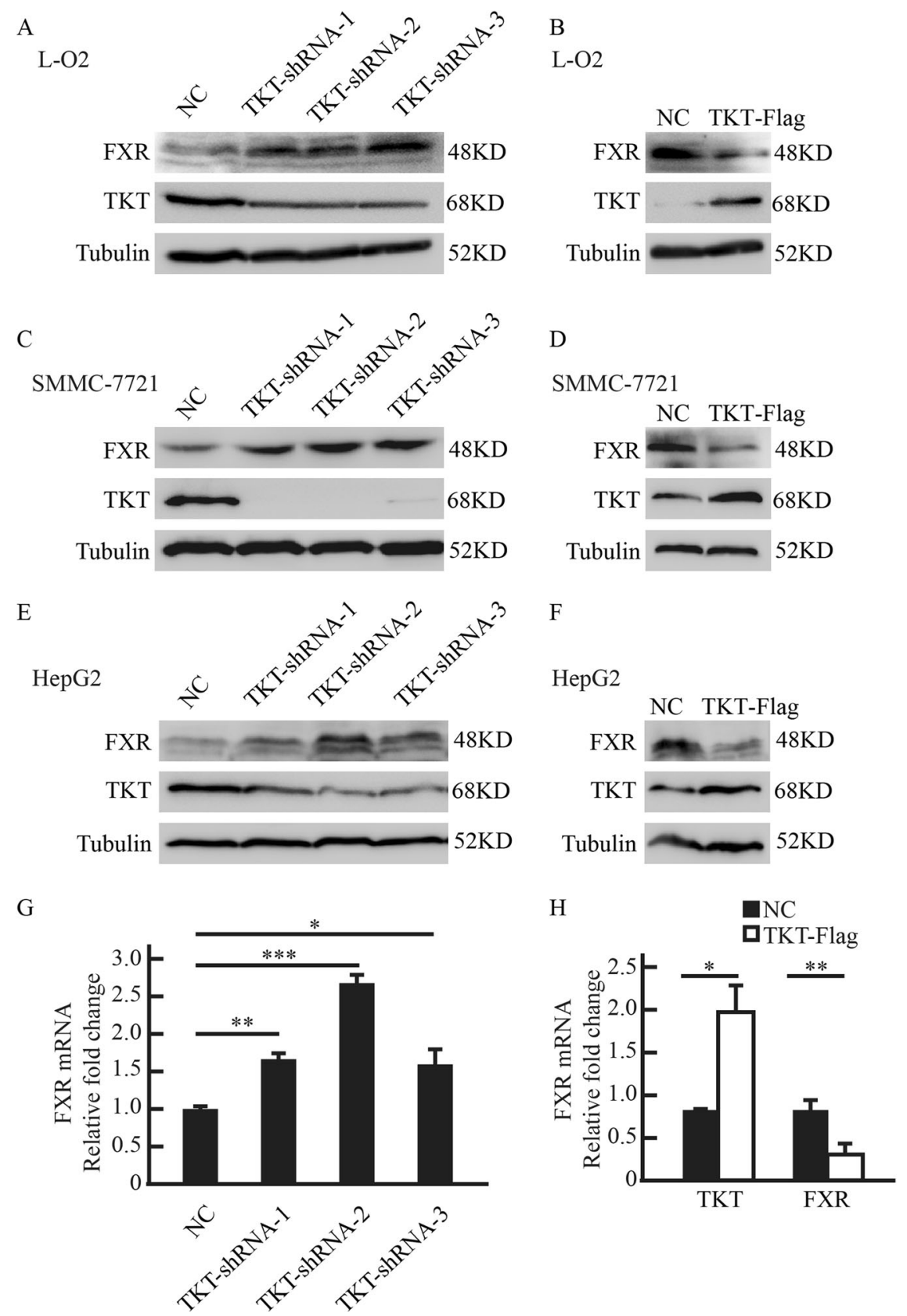

Fig. 2 The expression of FXR is negatively controlled by TKT. $\mathbf{a}, \mathbf{b}$ Western blot analysis shows that TKT negatively regulates FXR expression in L$\mathrm{O} 2$ cells. c, d Western blot analysis shows that TKT negatively regulates FXR expression in SMMC-7721 cells. e, $\mathbf{f}$ Western blot analysis shows that TKT negatively regulates FXR expression in HepG2 cells. $\mathbf{g}, \mathbf{h}$ QPCR analysis shows that TKT negatively regulates FXR mRNA level in SMMC-7721 cells. Data are presented as mean \pm squared error (SEM), the bar indicates the mean. Statistical significance was calculated using two-tailed unpaired $t$-test, ${ }^{*} p<$ $0.05,{ }^{* *} p<0.01,{ }^{* * *} p<0.001$.

with STAT1 in SMMC-7721 and HepG2 cells (Fig. 4b-e). Furthermore, coimmunoprecipitation results showed that TKT interacted with STAT1 simultaneously in the cytoplasm and nucleus (Fig. 4f). In addition, STAT1 overexpression increased the interaction in the nucleus (Fig. 4g).
STAT1 affected TKT nuclear localization and regulation of FXR promoter activity

To observe whether STAT1 affects TKT nuclear localization, we ectopically expressed STAT1 in SMMC-7721 cells and analyzed TKT subcellular localization using cell fractionation analysis. We found that overexpression of 

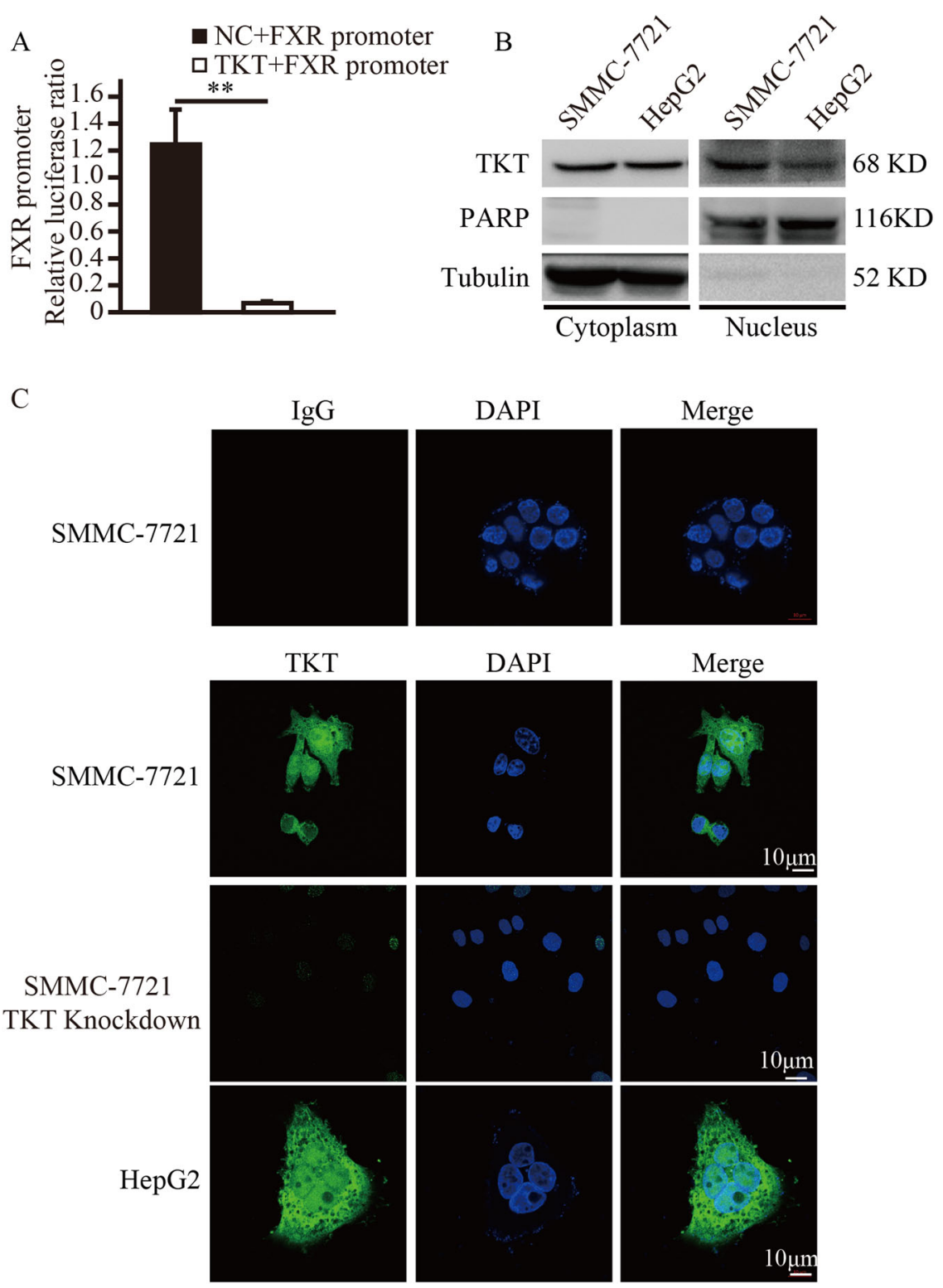

Fig. 3 TKT translocated into the nucleus and regulated the activity of the FXR promoter. a Luciferase activity analysis of the $2.5 \mathrm{~kb}$ FXR promoter at $48 \mathrm{~h}$ after the FXR promoter plasmid and empty vector or TKT-Flag expression plasmids were transfected into SMMC-7721 cells, Data are presented as mean \pm squared error (SEM), the bar indicates the mean. Statistical significance was calculated using two-tailed unpaired $t$-test, ${ }^{*} p<0.05$. b Nuclear and cytosolic fractionation analysis shows that TKT are localized in both the cytoplasm and nucleus of SMMC-7721 cells. Tubulin and PARP served as loading controls for the cytosolic and nuclear fraction, respectively. Every sample we loaded two lanes. c Immunofluorescent staining of endogenous TKT in SMMC-7721, SMMC-7721 cells with TKT shRNA and HepG2 cells.

STAT1 promoted TKT nuclear localization (Fig. 5a), and STAT1 shRNA-transfected SMMC-7721 cells had a reduced amount of TKT protein in the nuclear fractions (Fig. 5b), immunofluorescent staining further confirmed that STAT1 affected TKT nuclear localization (Fig. 5c and Supplementary Fig. S2).

We then analyzed whether TKT-STAT1 affects the activity of the FXR promoter. We transiently transfected the stable overexpression STAT1 cell line SMMC-7721 with the $2.5 \mathrm{~kb}$ FXR promoter linked to luciferase and analyzed luciferase activity $48 \mathrm{~h}$ later. The results showed that STAT1 inhibited the activity of the FXR promoter, but interestingly, luciferase activity was increased when the stable cell lines were transfected with TKT shRNA (Fig. 5d), and knockdown of STAT1 promoted luciferase activity in SMMC-7721 (Fig. 5e). Western blot further confirmed that STAT1 knockdown promoted FXR expression (Fig. 5f). These results suggested that STAT1 

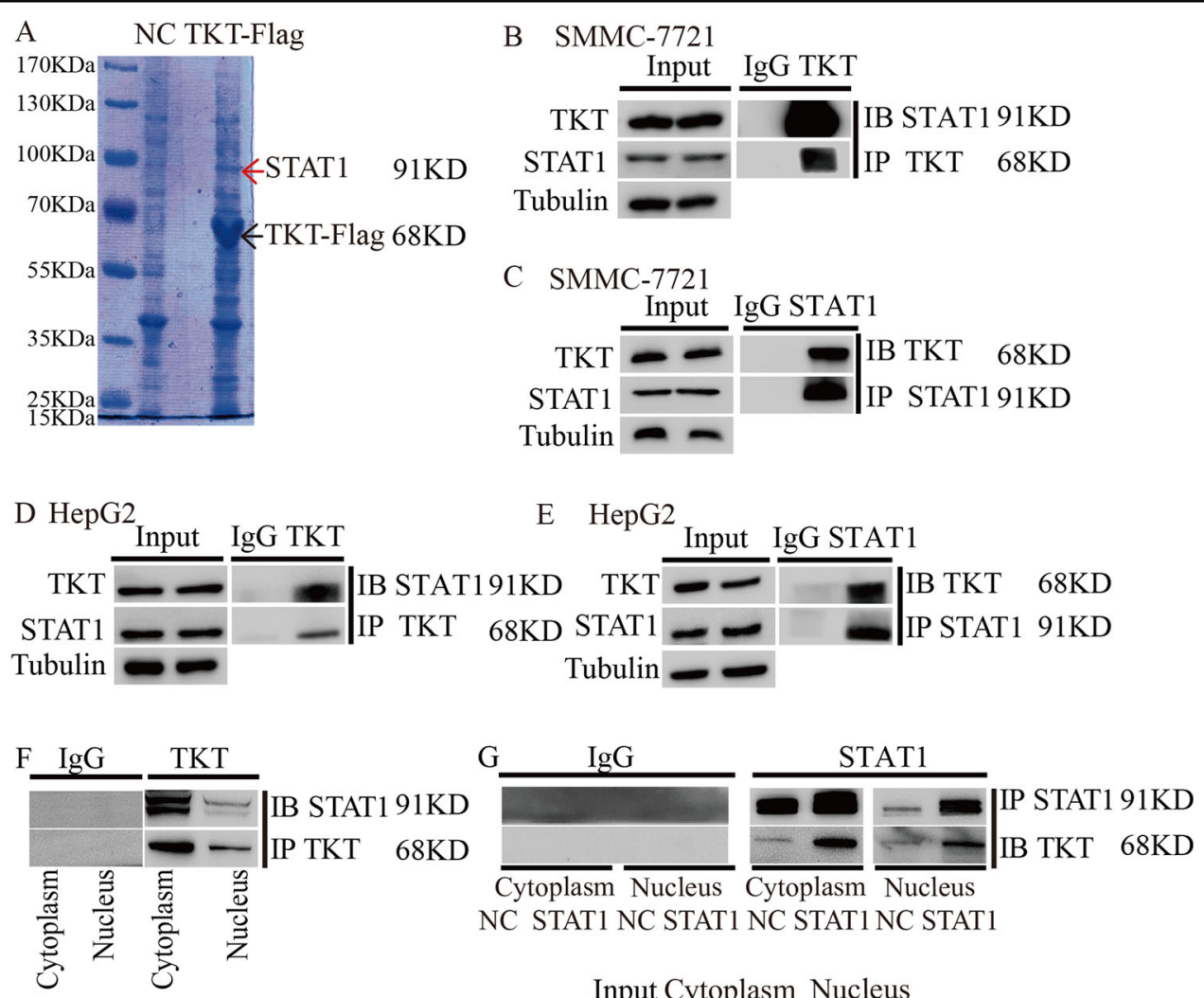

NC STAT1 NC STAT1 NC STAT1 NC STAT1
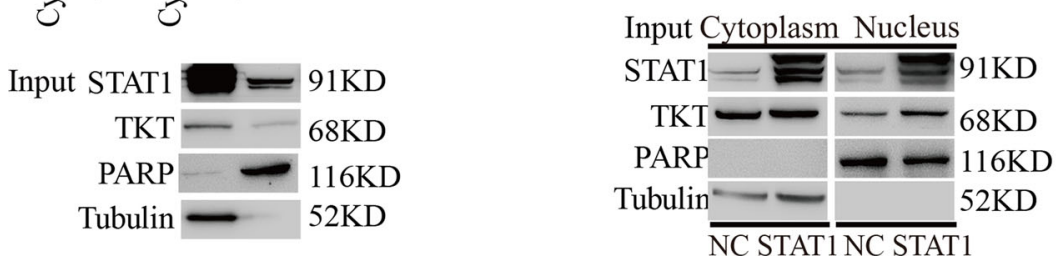

Fig. 4 TKT translocated into the nucleus through interacting with STAT1. a Silver-staining analysis of SMMC-7721 cell lysates immunoprecipitated using the anti-Flag antibody and normal rabbit lgG (NC). b, c Endogenous TKT coimmunoprecipitates with STAT1 in SMMC-7721 cells. d, e Endogenous TKT coimmunoprecipitates with STAT1 in HepG2 cells. f Endogenous TKT coimmunoprecipitates with STAT1 from the cytoplasm and nucleus of SMMC-7721 cells. $\mathbf{g}$ Endogenous TKT coimmunoprecipitates with STAT1 from the cytoplasm and nucleus of SMMC-7721 cells with aberrant expression of STAT1.

affected TKT nuclear localization and the regulation of TKT to FXR promoter.

\section{TKT inhibited FXR promoter activity by promoting the binding of HDAC3 to FXR promoter}

Given that TKT nuclear localization needed the help of STAT1, we next investigated how TKT regulates FXR promoter activity in the nucleus. Histone acetylation is one of the most important components of epigenetics. Histone deacetylases (HDACs) have emerged as major enzymes in the epigenetic regulation of gene expression by catalyzing the removal of acetyl groups, acetylation is one of the significant epigenetic markers associated with histones in the enhancers and promoters of genes, leading to chromatin remodeling and alterations in gene expres$\operatorname{sion}^{22}$. By using mass spectrometry analysis, we found that TKT could interact with HDAC3. We tried to verify the interaction between TKT, STAT1, and HDAC3 using coimmunoprecipitation (Fig. 6a).

After confirming that STAT1 affects TKT nuclear localization, we then investigated whether STAT1 affected the interaction between TKT and HDAC3 in the nucleus. Cell fractionation analysis showed that STAT1 overexpression increased the interaction of TKT and HDAC3 in the nucleus (Fig. 6b).

We postulated that TKT inhibited FXR expression through promoting the binding of HDAC3 to FXR promoter. We designed potential binding regions in the promoter of FXR, denoted as P1, P2, P3, P4, P5, P6, P7, and P8 (Fig. 6c). To determine whether TKT affected HDAC3's recruitment to the potential binding region in the FXR promoter, a chromatin immunoprecipitation (ChIP) assay was performed in SMMC-7721 TKT knockdown and overexpressing cells. ChIP-quantitative 


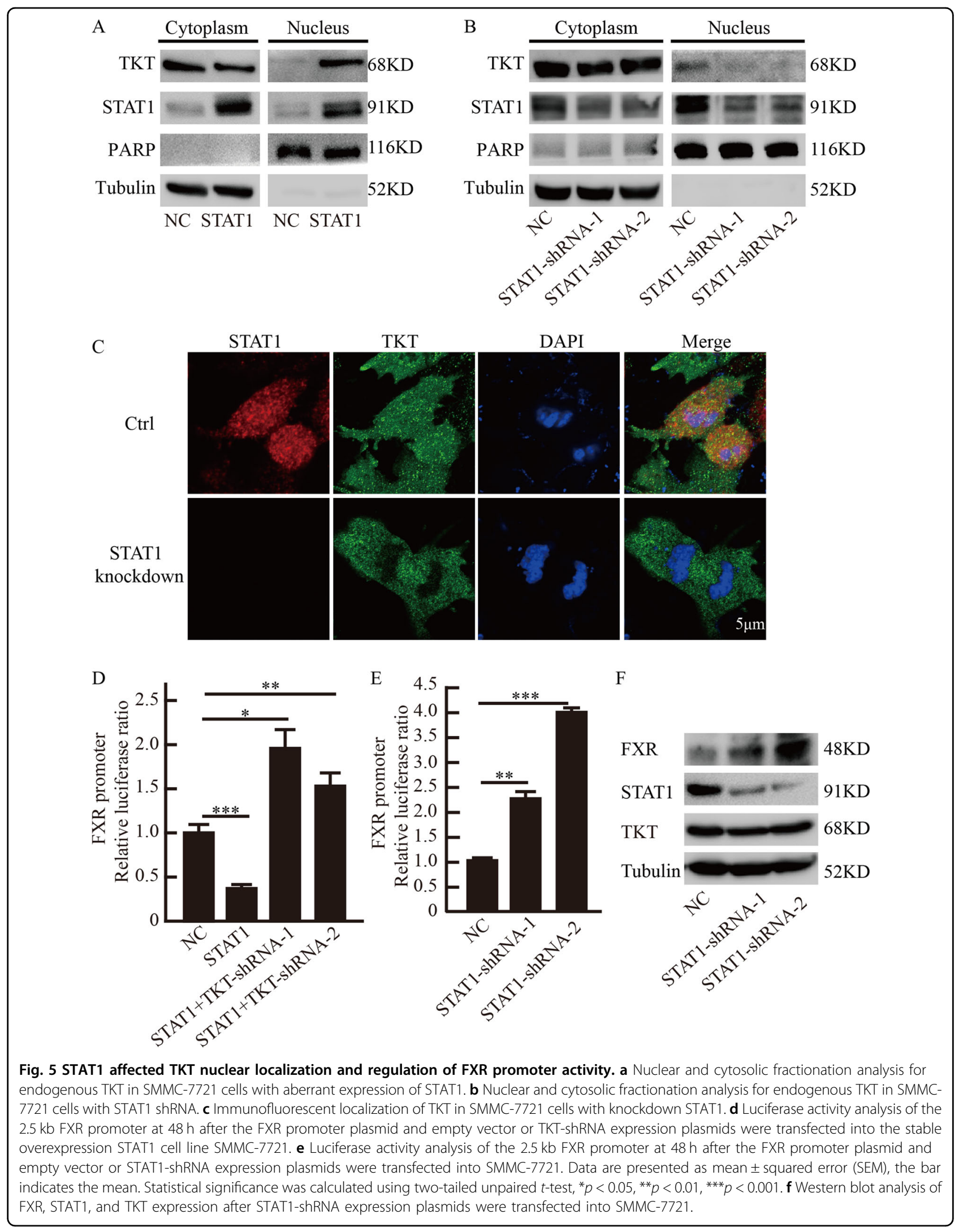




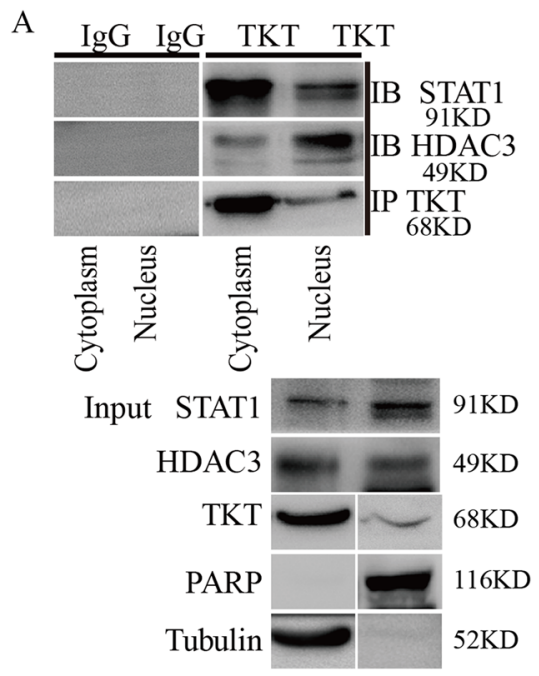

C

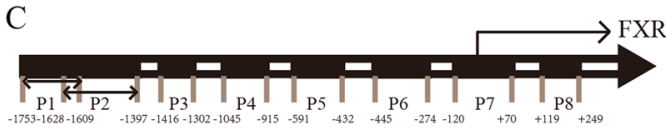

口IgG
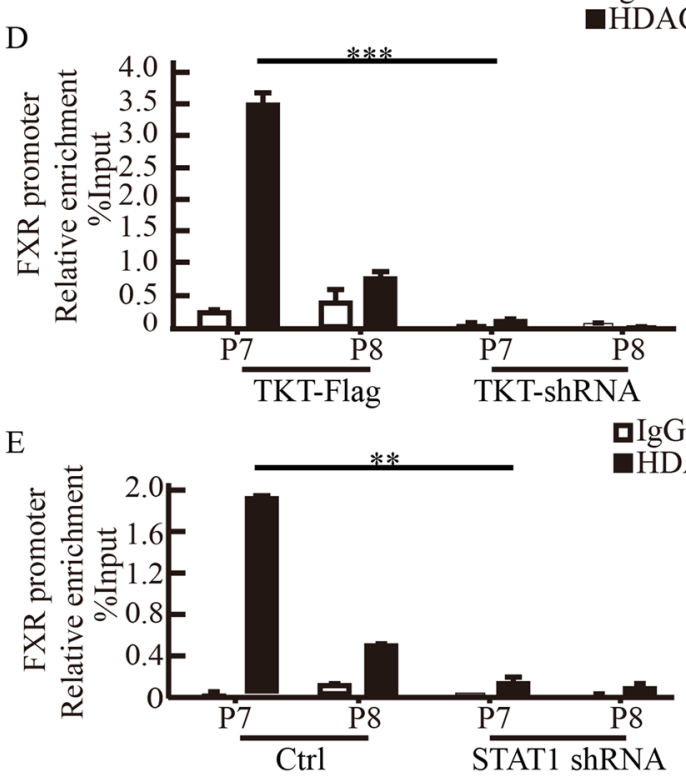

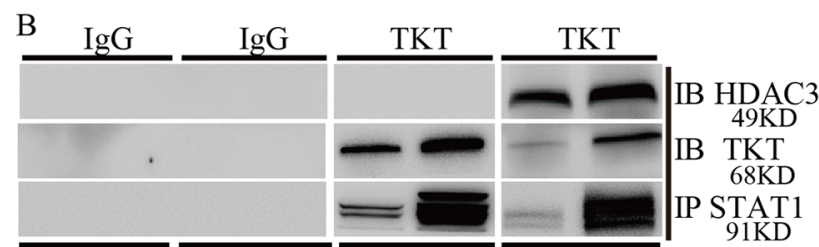

$\overline{\text { Cytoplasm }} \overline{\text { Nucleus }} \overline{\text { Cytoplasm Nucleus }}$

NC STAT1 NC STAT1 NC STAT1 NC STAT1

Input

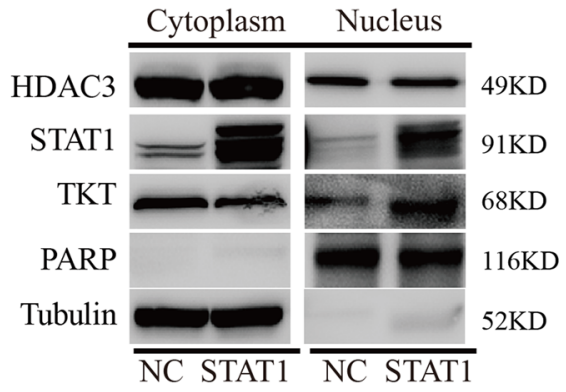

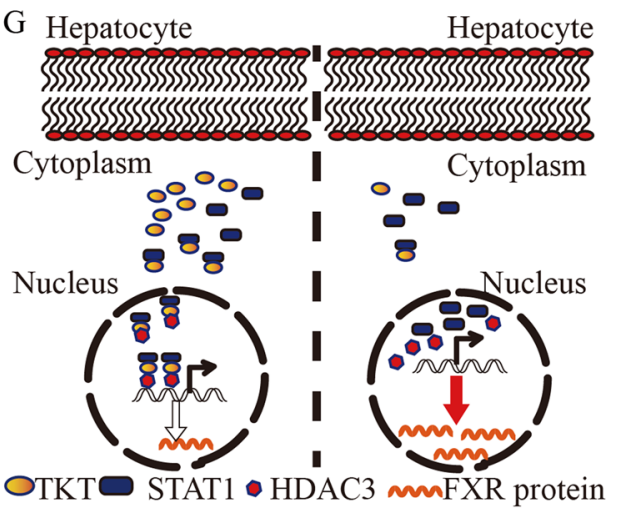

Fig. 6 TKT-STAT1 complex promotes the binding of HDAC3 to FXR promoter and inhibits FXR promoter activity. a Endogenous TKT coimmunoprecipitates with HDAC3 and STAT1 from the cytoplasm and nucleus of SMMC-7721 cells. b Endogenous TKT coimmunoprecipitates with HDAC3 and STAT1 from the nucleus and cytoplasm of SMMC-7721 cells ectopically expressing STAT1. c We designed potential binding regions in the promoter of FXR, denoted as P1, P2, P3, P4, P5, P6, P7, and P8. d ChIP analysis of SMMC-7721 cells using an anti-HDAC3 antibody or nonspecific lgG shows that the binding capacity of HDAC3 for FXR promoter was decreased when TKT was knocked down in SMMC-7721 cells. e ChIP analysis of SMMC-7721 cells using an anti-HDAC3 antibody or nonspecific IgG shows that the binding capacity of HDAC3 for FXR promoter was decreased when STAT1 was knockdown in SMMC-7721 cells. $\mathbf{f}$ ChIP analysis of SMMC-7721 cells using an anti-HDAC3 antibody or nonspecific lgG shows that the binding capacity of HDAC3 for FXR promoter was decreased when TKT was knockdown in SMMC-7721 cells. Data are presented as mean \pm squared error (SEM), the bar indicates the mean. Statistical significance was calculated using two-tailed unpaired $t$-test, ${ }^{*} p<0.05,{ }^{* *} p<0.01,{ }^{* * *} p<$ 0.001. g Schematic model of the role of TKT in regulating FXR expression. 
PCR results showed that HDAC3 mainly binds to the P7binding regions of the FXR promoter, and TKT promotes this binding (Fig. 6d). Furthermore, both STAT1 silencing and TKT silencing reduced the binding of HDAC3 to FXR promoter (Fig. 6e, f), western blot analysis showed that STAT1 and TKT are indeed knocked down (Supplementary Fig. S3). These results suggested that TKT inhibited FXR promoter activity by promoting the binding of HDAC3 to FXR promoter.

\section{Discussion}

Here we have identified a novel role of TKT in regulating bile acid in liver. Bile acids is one of the toxins to cause chronic inflammation and cell death ${ }^{23}$. Studies have indicated deoxycholic acid DCA can cause DNA damage, activation of hepatic stellate cells and the secretion of inflammatory tumor promoting factor. Intrahepatic cholestasis induces excessive fat accumulation, which could cause hepatocyte damage and inflammation, then promotes $\mathrm{HCC}^{24}$. Lithocholic acid (LCA) can stimulate the malignant proliferation of colon and breast cancer cells $^{25,26}$. Therefore, bile acids are tumor promoters and are involved in hepatocarcinogenesis. However, bile acid metabolism-target cancer therapy is still in its infancy.

FXR acts as a multifunctional cell protector in the liver through regulating the homeostasis of bile acids critically. FXR overexpression has been demonstrated to repress cancer cell proliferation ${ }^{27,28}$. It is has been shown that mice that have high levels of FXR do not develop liver cancer after treatment with $\mathrm{DEN}^{29}$. Therefore, FXR is a tumor suppressor.

Metabolic enzymes play important roles in tumorigenesis. TKT catalyzes several key reactions of the nonoxidative phase of PPP, allowing cells to adapt to a variety of metabolic needs under different conditions ${ }^{30}$. It has been demonstrated that TKT promoting breast cancer metastasis through regulating the metabolic switch ${ }^{31}$. In several human cancers, such as HCC and metastatic peritoneal implants of ovarian cancer, TKT expression is increased significantly ${ }^{4,18}$. Furthermore, TKT levels is closely related to chemotherapeutic resistance of tumor $^{32-34}$. Of note, it has been shown that long noncoding RNA TSLNC8 blocks tumor growth by reducing the interaction between TKT and STAT3 and inhibiting IL-6/STAT3 signaling pathway ${ }^{35}$. Therefore, TKT is a key factor involved in tumorigenesis.

Previous studies have focused on TKT as a metabolic enzyme regulating the PPP pathway to affect tumor cell proliferation. In our study, we have made a metabolomics analysis of livers of WT and TKT $T^{f l f l}$ Alb-cre mice. The results showed that compared with littermates, lacking of TKT altered bile acid metabolism, primary and second bile acid were decreased. This is an interesting phenomenon, which means that TKT may affect the development of HCC through regulating intrahepatic bile acids. During liver injury induced by DEN, cholestasis exacerbate liver inflammation, however, mice that have high levels of FXR expression do not develop liver cancer after treatment with $\mathrm{DEN}^{29}$.

In the current study, TKT deficiency in hepatocytes alleviated bile acids level in liver. Accordingly, our results showed that TKT was present not only in the cytosolic fraction but also in the nuclear fraction, and that TKT needs to interact with STAT1 to translocate into the nucleus, where with the complex promotes the binding of HDAC3 to FXR promoter, eventually inhibiting the expression of FXR (Fig. 6g). Taken together, we identified a novel role of TKT in regulating intrahepatic bile acid. Indeed, reduced cholestasis in hepatocytes offers an opportunity of HCC therapy. Therefore, targeting the metabolic enzyme TKT is an attractive strategy for bile acid metabolism in livers and cancer therapy.

\section{Acknowledgements}

This work is supported by funding from the National Natural Science Foundation of China (No. 81672845, and 81872304); the Science and Technology Project of Xuzhou (KC17106); Jiangsu Planned Projects for Postdoctoral Research Funds (No. 2018K246C); China Projects for Postdoctoral Research Funds (2019M651968); The Research Foundation of Xuzhou Medical University (No.53681624); Jiangsu Province Natural Science Foundation (No. BK20170266, BK20150216).

\section{Author details \\ ${ }^{1}$ Cancer Institute, Xuzhou Medical University, 221002 Xuzhou, Jiangsu, China. ${ }^{2}$ Jiangsu Center for the Collaboration and Innovation of Cancer Biotherapy, Xuzhou Medical University, 221002 Xuzhou, Jiangsu, China. ${ }^{3}$ Department of Biochemistry and Molecular Cell Biology, Shanghai Key Laboratory for Tumor Microenvironment and Inflammation, Key Laboratory of Cell Differentiation and Apoptosis of Chinese Ministry of Education, Shanghai Jiao Tong University School of Medicine, 200025 Shanghai, China. ${ }^{4}$ Affiliated Hospital of Xuzhou Medical University, 221002 Xuzhou, Jiangsu, China. ${ }^{5}$ Center of Clinical Oncology, Affiliated Hospital of Xuzhou Medical University, 221002 Xuzhou, Jiangsu, China}

Conflict of interest

The authors declare that they have no conflict of interest.

\section{Publisher's note}

Springer Nature remains neutral with regard to jurisdictional claims in published maps and institutional affiliations.

Supplementary Information accompanies this paper at (https://doi.org/ 10.1038/s41419-020-2225-6).

Received: 25 June 2019 Revised: 27 December 2019 Accepted: 28 December 2019

Published online: 16 January 2020

\section{References}

1. Chen, W. et al. Cancer statistics in China, 2015. Cancer J. Clin. 66, 115-132 (2016).

2. El-Serag, H. B. Epidemiology of viral hepatitis and hepatocellular carcinoma. Gastroenterology 142, 1264-1273 e1261 (2012).

3. Hertl, M. \& Cosimi, A. B. Liver transplantation for malignancy. Oncologist 10 269-281 (2005). 
4. Xu, I. M. et al. Transketolase counteracts oxidative stress to drive cancer development. Proc. Natl Acad. Sci. USA 113, E725-E734 (2016).

5. Li, T. \& Chiang, J. Y. Bile acid signaling in metabolic disease and drug therapy. Pharm. Rev. 66, 948-983 (2014)

6. Trauner, M. \& Boyer, J. L. Bile salt transporters: molecular characterization, function, and regulation. Physiol. Rev. 83, 633-671 (2003).

7. Zollner, G. \& Trauner, M. Mechanisms of cholestasis. Clin. Liver Dis. 12, 1-26 (2008). vii.

8. Huang, X. F., Zhao, W. Y. \& Huang, W. D. FXR and liver carcinogenesis. Acta Pharm. Sin. 36, 37-43 (2015).

9. Goodwin, B. et al. A regulatory cascade of the nuclear receptors FXR, SHP-1, and LRH-1 represses bile acid biosynthesis. Mol. Cell 6, 517-526 (2000).

10. Inagaki, $\mathrm{T}$. et al. Fibroblast growth factor 15 functions as an enterohepatic signal to regulate bile acid homeostasis. Cell Metab. 2, 217-225 (2005).

11. Fiorucci, S. et al. The nuclear receptor SHP mediates inhibition of hepatic stellate cells by FXR and protects against liver fibrosis. Gastroenterology 127, 1497-1512 (2004).

12. Li, J. et al. Inhibition of endothelin-1-mediated contraction of hepatic stellate cells by FXR ligand. PLoS One 5, e13955 (2010).

13. Wang, Y. D. et al. Farnesoid $X$ receptor antagonizes nuclear factor kappaB in hepatic inflammatory response. Hepatology 48, 1632-1643 (2008).

14. Li, T. \& Apte, U. Bile acid metabolism and signaling in cholestasis, inflammation, and cancer. Adv. Pharm. 74, 263-302 (2015).

15. Kim, I. et al. Spontaneous hepatocarcinogenesis in farnesoid $X$ receptor-null mice. Carcinogenesis 28, 940-946 (2007).

16. Yang, F. et al. Spontaneous development of liver tumors in the absence of the bile acid receptor farnesoid X receptor. Cancer Res. 67, 863-867 (2007).

17. Xu, Z. P., Wawrousek, E. F. \& Piatigorsky, J. Transketolase haploinsufficiency reduces adipose tissue and female fertility in mice. Mol. Cell Biol. 22, 6142-6147 (2002)

18. Ricciardelli, C. et al. Transketolase is upregulated in metastatic peritoneal implants and promotes ovarian cancer cell proliferation. Clin. Exp. Metastasis 32, 441-455 (2015).

19. Wang, J. et al. Inhibition of transketolase by oxythiamine altered dynamics of protein signals in pancreatic cancer cells. Exp. Hematol. Oncol. 2, 18 (2013).

20. Huang, Q. et al. Metabolic characterization of hepatocellular carcinoma using nontargeted tissue metabolomics. Cancer Res. 73, 4992-5002 (2013).
21. Li, M. et al. Transketolase deficiency protects the liver from DNA damage by increasing levels of ribose 5-phosphate and nucleotides. Cancer Res. 79, 3689-3701 (2019)

22. McQuown, S. C. \& Wood, M. A. HDAC3 and the molecular brake pad hypothesis. Neurobiol. Learn Mem. 96, 27-34 (2011).

23. Fan, M., Wang, X., Xu, G., Yan, Q. \& Huang, W. Bile acid signaling and liver regeneration. Biochim Biophys. Acta 1849, 196-200 (2015).

24. Sun, L. et al. Bile acids promote diethylnitrosamine-induced hepatocellular carcinoma via increased inflammatory signaling. Am. J. Physiol. Gastrointest. Liver Physiol. 311, G91-G104 (2016).

25. Baker, P. R. et al. Bile acids influence the growth, oestrogen receptor and oestrogen-regulated proteins of MCF-7 human breast cancer cells. Br. J. Cancer 65, 566-572 (1992).

26. Flynn, C. et al. Deoxycholic acid promotes the growth of colonic aberrant crypt foci. Mol. Carcinog. 46, 60-70 (2007).

27. Deuschle, U. et al. FXR controls the tumor suppressor NDRG2 and FXR agonists reduce liver tumor growth and metastasis in an orthotopic mouse xenograft model. PLoS One 7, e43044 (2012).

28. Modica, S., Murzilli, S., Salvatore, L., Schmidt, D. R. \& Moschetta, A. Nuclear bile acid receptor FXR protects against intestinal tumorigenesis. Cancer Res. $6 \mathbf{8}$ 9589-9594 (2008).

29. Jiang, $Y$. et al. Farnesoid $X$ receptor inhibits gankyrin in mouse livers and prevents development of liver cancer. Hepatology 57, 1098-1106 (2013).

30. Horecker, B. L. The pentose phosphate pathway. J. Biol. Chem. 277 47965-47971 (2002)

31. Tseng, C. W. et al. Transketolase regulates the metabolic switch to control breast cancer cell metastasis via the alpha-Ketoglutarate signaling pathway. Cancer Res. 78, 2799-2812 (2018).

32. Semenza, G. L. HIF-1 mediates metabolic responses to intratumoral hypoxia and oncogenic mutations. J. Clin. Invest. 123, 3664-3671 (2013).

33. Shukla, S. K. et al. MUC1 and HIF-1alpha signaling crosstalk induces anabolic glucose metabolism to impart gemcitabine resistance to pancreatic cancer. Cancer Cell 32, 392 (2017).

34. Zhao, F. et al. Imatinib resistance associated with BCR-ABL upregulation is dependent on HIF-1alpha-induced metabolic reprograming. Oncogene $\mathbf{2 9}$ 2962-2972 (2010).

35. Zhang, J. et al. Long noncoding RNA TSLNC8 is a tumor suppressor that inactivates the interleukin-6/STAT3 signaling pathway. Hepatology 67, 171-187 (2018). 